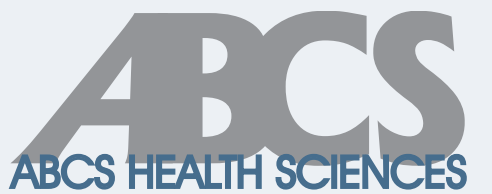

How to cite this article: Oliveira et al. Association of clinical epidemiological factors to polypharmacy among patients with multiple sclerosis: real-life data. ABCS Health Sci. 2021;46:e021212. https://doi. org/10.7322/abcshs.2020137.1899

Received: Sep 11, 2020

Approved: Oct 15, 2020

Corresponding author: Vanessa Terezinha Gubert - Faculdade de Ciências

Farmacêuticas, Alimentos e Nutrição, Universidade Federal de Mato Grosso do Sul - Avenida Costa e Silva, s/n - Cidade Universitária - CEP: 79070-900 - Campo Grande (MS), Brazil -

E-mail: vanessa.matos@ufms.br

Declaration of interests: nothing to declare Funding: CAPES, UFMS

This is an open access article distributed under the terms of the Creative Commons Attribution License

(C) 2021 Oliveira et al.

\title{
Association of clinical epidemiological factors to polypharmacy among patients with multiple sclerosis: real-life data
}

\author{
Vanessa Marcon de Oliveira ${ }^{1}$, Cinthia Caldas Rios ${ }^{2}$, Vanessa Terezinha Gubert ${ }^{1,2}$, \\ Cristiane Munaretto Ferreira1 ${ }^{1}$, Erica Freire de Vasconcelos-Pereira1, \\ Mônica Cristina Toffoli-Kadri², Maria Tereza Ferreira Duenhas Monreal ${ }^{1,2}$ \\ ${ }^{1}$ Farmácia Escola Profa Ana Maria Cervantes Baraza, Faculdade de Ciências Farmacêuticas, \\ Alimentos e Nutrição, Universidade Federal de Mato Grosso do Sul (UFMS) - Campo Grande (MS), \\ Brazil. \\ ${ }^{2}$ Pós-Graduação em Ciências Farmacêuticas, Faculdade de Ciências Farmacêuticas, Alimentos e \\ Nutrição, UFMS - Campo Grande (MS), Brazil.
}

\begin{abstract}
Introduction: Treatment for multiple sclerosis should focus on relapse prevention and treatment, as well as symptom and disease progression control, which require the use of multiple medications. Objective: To evaluate the association of polypharmacy and related clinical, epidemiological factors in multiple sclerosis patient cohorts. Methods: It was conducted a prospective study of multiple sclerosis patients that held a prescription of disease-modifying drugs between January and December 2017. The medications were analyzed and classified as either long-term or as-needed medications for therapeutic objective and prescription status purposes. Results: During 2017, 124 patients were attended, 106 were eligible for the study, and 81 agreed to participate. The average age was $40.95 \pm 11.69$ years. The disease duration varied between 6 months and 30 years, with a median of 7 years. More than half of the multiple sclerosis patients suffered from comorbidities (54.32\%), and 76.54\% were categorized as polypharmacy. The comparison of polypharmacy between the groups yielded significant differences for comorbidities and employment status and regarding age between patients with polypharmacy and patients without polypharmacy of long-term medications. Conclusion: The age of the patient and the presence of comorbidities are important factors related to polypharmacy.
\end{abstract}

Keywords: multiple sclerosis; pharmacy, patient care; comorbidity; drug combinations; medication systems.

\section{INTRODUCTION}

It is estimated that 2.5 million people worldwide have been diagnosed with multiple sclerosis (MS), with a global prevalence in 2013 of 33 per 100,000 inhabitants ${ }^{1}$. This prevalence varies considerably between countries ${ }^{2}$. In Brazil, it is estimated that the prevalence of MS ranges from 0.75 to 30.7 cases per 100,000 inhabitants, depending on the characteristics of the population studies performed ${ }^{3}$. 
MS is a progressive disease, which is difficult to predict, and can result in disability and/or physical, mental, and social limitations. Study of the emotional status of the individual, which is closely related to the evolution of MS, may assist when monitoring treatment efficacy and could help to achieve better results ${ }^{4}$.

As there is no cure, treatment for MS should focus on relapse prevention and treatment, as well as symptom and disease progression control ${ }^{5}$, which requires the use of multiple medications ${ }^{6}$. In this sense, the available drugs (including complementary drugs and alternative therapies) have had significant beneficial effects on the quality of life of patients ${ }^{7}$.

The most common definition of polypharmacy is the concurrent use of five or more medication ${ }^{8}$. The increasing frequency of polypharmacy has been observed in the general population. Older people are particularly affected because they are more likely to have comorbidities and take more medication daily. Ignoring the polypharmacy factor can lead to readmissions, severe drug interactions ${ }^{9}$, poor adherence, cognitive decline, increased costs, and risk of side effects ${ }^{10}$. To date, there are few studies on polypharmacy in MS ${ }^{11-14}$.

This study aimed to evaluate the association of clinical, epidemiological factors and polypharmacy in an MS patient cohort.

\section{METHODS}

\section{Study design and population}

It was conducted a prospective study of an MS patient cohort that held a prescription of disease-modifying drugs (DMDs) during 2017 and received treatment through the specialized component of the Pharmaceutical Assistance of the Health Secretariat of Mato Grosso do Sul (SES-MS).

Patients diagnosed with relapsing-remitting MS (RRMS), as defined using the criteria of McDonald in 2010 and G-35 classification in ICD-10, were included in the study. RRMS patients represent the most common subtype worldwide, and the clinical protocol is already approved for the treatment of this subtype in Brazil. Patients with three or more consecutive months without drug withdrawal were considered inactive and excluded from the study.

Ethics approval was granted by the Research Ethics Committee of Universidade Federal de Mato Grosso do Sul (no. 1.777.902). All participants signed an informed consent form prior to the beginning of the study, according to the Declaration of Helsinki.

\section{Data collection}

Clinical epidemiological and therapeutic data were collected from medical records and the Computerized Management and Monitoring System for Exceptional Medicines (SISMEDEX). Data was also obtained through structured interviews.
Epidemiological data included gender, age, marital status, education, and employment status. The types of clinical data collected were degree of disability, using the Kurtzke Expanded Disability Status Scale (EDSS); disease duration, measured from the initial diagnosis; and the presence of comorbidities, comparing patients with secondary illnesses (PwSI) and patients without secondary illnesses (Pw/oSI).

Pharmacological data included the active principle of the drug preparations and indications. All the drugs taken by the patients were included in the data analysis.

\section{Drug analysis}

The medications were analyzed and classified for therapeutic objective and prescription status purposes. For classification, drugs were divided into long-term and as-needed medications. Long-term medications refer to those that are taken daily or at regular intervals to treat long-term health problems, and as-needed medications refer to those taken at not regular intervals to treat acute or eventual health problems.

To assess the therapeutic objective, drugs were grouped into medicines specifics for MS and those to treat other health problems. Specific symptomatic drugs are those used to treat or alleviate symptoms specific to MS. For prescription status, drugs were classified as either prescription-only or over-the-counter (OTC) medications.

\section{Polypharmacy and secondary illnesses}

There are several definitions of polypharmacy. In this study, we adopted the definition of Richardson et al. ${ }^{8}$ when categorizing patients with polypharmacy $(\mathrm{PwP})$ (those concurrently using five or more medications) and patients without polypharmacy (Pw/oP) (those using fewer than five medications). Polypharmacy was analyzed in two ways: (a) by calculating the sum of all long-term and as-needed medications and (b) by the number of long-term medications alone.

The definition of comorbidities was adopted according to Laroni et al. ${ }^{15}$, while the recommendations for observational studies on comorbidities in MS followed the established by Marrie et al. ${ }^{16}$. The PwSI was defined based on patient records and interviews and the opinion of a physician.

\section{Statistical analysis}

Data were analyzed using SPSS 23 and tested for homogeneity of variances (Levene's test).

It was used two-sample, two-tailed Student's t-tests, Fisher's exact tests, and Chi-square tests to compare the different patient groups ( $\mathrm{PwP}$ and $\mathrm{Pw} / \mathrm{oP})$.

Associations between polypharmacy (defined by the total number of drugs used) and clinical, epidemiological variables (gender, age, highest educational attainment, partnership status, 
employment status, comorbidities, disease duration, and EDSS score) were examined using a multivariable logistic regression model. The level of significance was established at $\alpha=0.05$. False discovery rate (FDR) adjusted p-values were used to mitigate alpha inflation in multiple testing. The pairwise interdependencies between several variables were identified using the analysis of Pearson's correlation coefficients.

The comparison between the means of medication used by patients according to drug classification was analyzed using MannWhitney U tests.

\section{RESULTS}

During 2017, 160 patients were assisted by CEAF in the state of Mato Grosso do Sul. Of these, 124 were treated at the Professor Ana Maria Cervantes Baraza School of Pharmacy, 106 were eligible to take part in the study, and 81 agreed to participate.

The average age was $40.95 \pm 11.69$ years (range: 18 to 70 years). No illiterate patients were identified. The disease time occurrence varied between 6 months and 30 years, with a median time of 7 years. The median EDSS score was 4.0, with individual scores ranging between 0.0 and 7.5.

Comorbidities were identified in more than half of the MSassisted patients (54.32\%), with psychiatric disorders being the most common associated conditions (20.98\%). Diseases such as hypothyroidism $(14.81 \%)$ and systemic arterial hypertension (11.11\%) were also identified.

In the analysis of the examined patients, $76.54 \%(62 / 81)$ were categorized as PwP. Of those 62 patients, 20.97\% (13/62) were aged between 20 and 29 years, $51.61 \%(32 / 62)$ had a higher educational level, and $35.48 \%(22 / 62)$ were economically active. The clinical, epidemiological factors related to polypharmacy in this MS patient cohort were age, comorbidities, and employment status.

The comparison of the polypharmacy between the groups yielded significant differences for comorbidities $(\mathrm{p}=0.0013$ and $\mathrm{p}=0.0001$ ), employment status ( $\mathrm{p}=0,013$ and $\mathrm{p}=0.007$ ), and age between PwP and Pw/oP for long-term medications $(\mathrm{p}=0.001)$. There was no significant difference between the groups regarding EDSS scale values or time since diagnosis (Table 1).

Overall, the average number of medications taken by the 81 patients was 6.15 (SD 2.52), with the minimum being 1 medication and the maximum 14 . The total number of medications taken by the patients was 498 , including repetitions. Of these, $88.75 \%$ were used by PwP. The most common treatments used by patients were vitamin $\mathrm{D}$ as an adjuvant to MS treatment (87.7\%), analgesics (87.7\%), antidepressants $(32.1 \%)$, and dietary supplements (18.5\%). Fampridine was used by $4.9 \%(4 / 81)$ of the patients as a symptomatic treatment for a walking disability.
There was significant group (long-term and total medications) differences concerning polypharmacy in the frequency of treatment use (Table 2).

The average number of medications taken by the group with polypharmacy (7.12) was two to three times higher than that by the group without polypharmacy (2.95). When analyzing patients using long-term medications, we find that $22.22 \%$ (18/81) were those with polypharmacy. The overall average number of longterm treatments was 3.12 medications per patient; broken down by group, the averages were $6.39(\mathrm{PwP})$ and $2.19(\mathrm{Pw} / \mathrm{oP})$.

There was a significant difference between the $\mathrm{PwP}$ and $\mathrm{Pw} / \mathrm{oP}$ groups in the number of medications taken, according to different categories. When evaluating polypharmacy with long-term medications, we find that only the categories of pro re nata $(\mathrm{PRN})$ and symptomatic medications showed no significant difference (Table 3).

Significant relationships between variables were observed using Pearson's correlation coefficients, as shown in Figure 1.

\section{DISCUSSION}

The average age of the study population was comparable with that in other studies on polypharmacy in $\mathrm{MS}^{12-14}$. Economically inactive patients were the majority, which is similar to the results of studies conducted in Germany ${ }^{6,17}$ and is likely due to the limiting nature of the disease ${ }^{18}$ that, even with low EDSS values, may cause an inability to work ${ }^{17}$.

Although the degree of disability was moderate, we observed a high number of patients with an EDSS value greater than 5.0, which can be explained by the fact that many patients had been diagnosed several years before. Most Brazilian cohorts reported EDSS values below $4.0^{3,19}$, similar to values reported in other international studies ${ }^{6,17,20}$.

In the analysis of all drugs, we observed that polypharmacy was present in $76.5 \%$ of patients. When considering the second classification that excluded PRN medications, the polypharmacy rate was $22.2 \%$. These results differ from the Literature ${ }^{6,11-14,17}$. The reduced frequency of polypharmacy in long-term medication and all-drug analyses may be explained by the large number of patients using drugs classified as PRN, especially analgesics, which are used to relieve symptoms caused by an adverse reaction to MS medication. Often patients use analgesic drugs of different mechanisms of action to reduce their symptoms.

Studies on polypharmacy in MS patients are still scarce $e^{6,11-14,17,20}$. Previous studies have analyzed the effect of antiepileptic and antidepressant drugs on fatigue and cognitive ability, quality of life, and relapse rate ${ }^{11,13,14}$. Our study, like others published recently ${ }^{6,17}$, investigates the use of all medications by patients with MS and analyzes the clinical and epidemiological factors related to polypharmacy in those cases. 
Table 1: Clinical epidemiological data of the examined MS patient cohort with prescription of disease-modifying therapy in 2017.

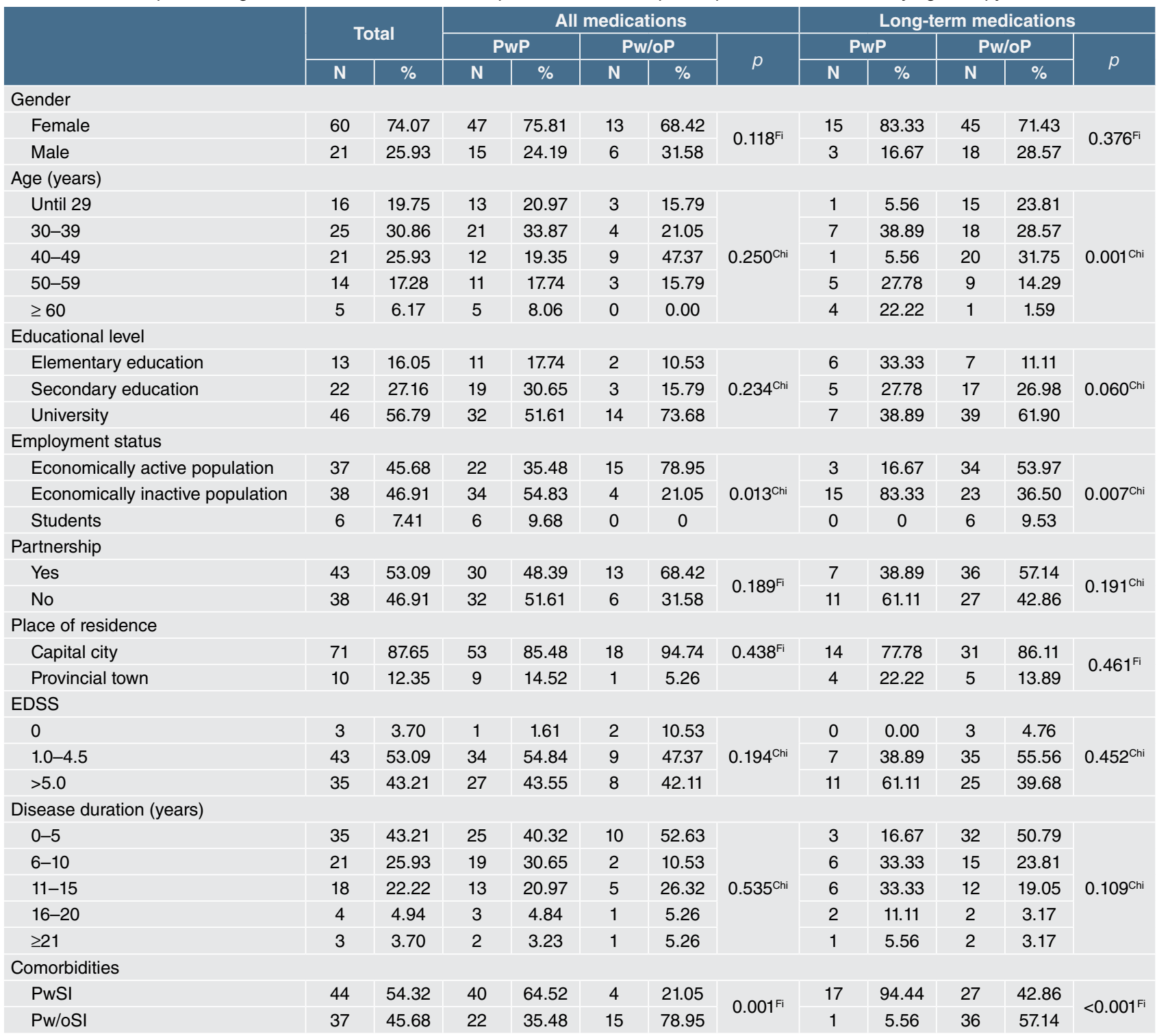

MS: Multiple sclerosis; N: Number of patients; PwP: Patients with polypharmacy; Pw/oP: Patients without polypharmacy; EDSS: Expanded Disability Status Scale; Fi: Fisher's exact test; Chi: Chi-square test.

The use of multiple medicines to treat chronic diseases is common in the older population with comorbidity. The health burden of multimorbidity is expected to rise significantly as the result of an aging population ${ }^{21}$. Polypharmacy has a direct correlation with comorbidity since the existence of comorbidity usually requires additional treatments, resulting in an increase in the occurrence of polypharmacy. Among PwP, comorbidities were almost twice as prevalent, and polypharmacy was approximately seven times higher for patients who had associated secondary diseases ( $p=0.0013$; odds ratio 6.82). A 2015 meta-analysis review found that the five most common comorbidities in MS are depression, anxiety, hypertension, hyperlipidemia, and chronic lung disease. This same study identified that hypothyroidism is among the most prevalent autoimmune diseases ${ }^{22}$. In addition to the presence of comorbidities inducing polypharmacy, some drugs used to treat MS can also cause secondary diseases and side effects and thus require drug interventions ${ }^{23,24}$.

A more detailed analysis of the medication used revealed that PwP used, on average, more drugs than those without polypharmacy. DMDs did not contribute to this quantitative drug difference between the two groups because MS immunotherapy is usually maintained by monotherapy ${ }^{25}$.

The analysis of drugs used by MS patients evidenced that vitamin $\mathrm{D}$, antidepressants, and dietary supplements were the most frequently used, followed by antispastic and antihypertensive drugs. Since DMDs are the basis of treatment for MS, as they 
Table 2: Comparison of treatments used between the groups concerning polypharmacy.

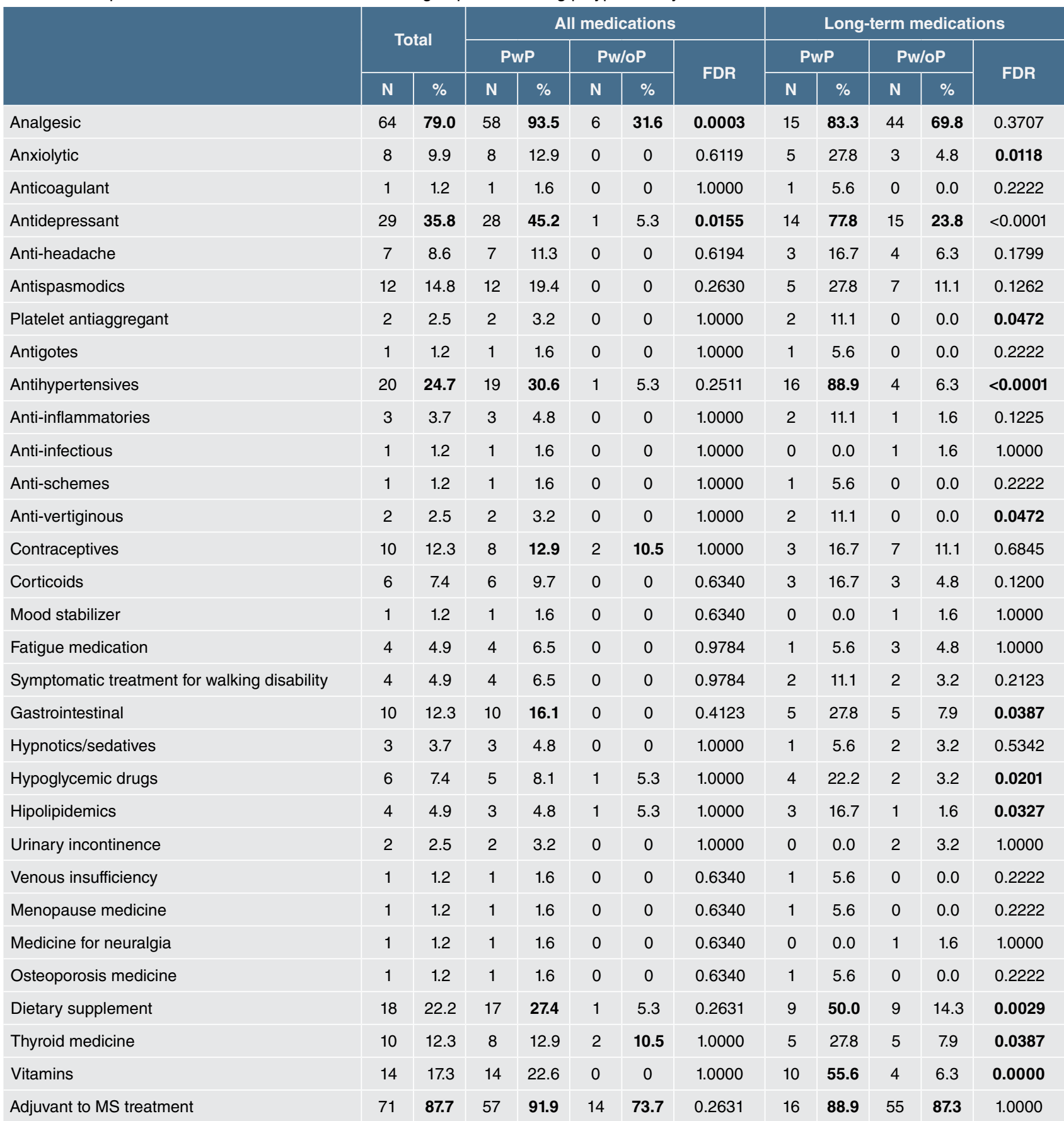

PwP: Patients with polypharmacy; Pw/oP: Patients without polypharmacy; FDR: False discovery rate; MS: Multiple sclerosis

prevent relapses and delay disease progression, all patients used a specific medication to treat $\mathrm{MS}^{26}$.

The therapeutic use of vitamin D in the treatment of MS is a controversial subject that is of interest to doctors, researchers, and patients. In clinical practice, there is no solid scientific evidence to justify its use in monotherapy in the treatment of MS. An in vivo study found that $\mathrm{T}$-cell reactivity was suppressed by vitamin $\mathrm{D}$ at serum 25(OH)D concentrations above $40 \mathrm{ng} / \mathrm{ml}$ and could cause modulating action on the immune system. This, in turn, could contribute to a reduction in the inflammatory processes in $\mathrm{MS}^{27}$. In this context, the use of vitamin D supplements, at doses capable of maintaining a patient's serum level between 40 and $100 \mathrm{ng} / \mathrm{ml}$, could be beneficial in reducing the symptoms of MS. It is noteworthy that serum levels above $100 \mathrm{ng} / \mathrm{ml}$ are considered toxic and should be avoided ${ }^{28}$. However, there is a lack of consensus on the subject, which is evidenced by a meta-analysis study 
Table 3: Comparison between the means of medication used by patients, according to drug classification

\begin{tabular}{|c|c|c|c|c|c|c|c|c|c|c|c|c|}
\hline \multirow{3}{*}{$\begin{array}{l}\text { Drug } \\
\text { classification }\end{array}$} & \multirow{2}{*}{\multicolumn{2}{|c|}{ Total }} & \multicolumn{5}{|c|}{ Polypharmacy } & \multicolumn{5}{|c|}{ Polypharmacy with long-term medications } \\
\hline & & & \multicolumn{2}{|c|}{ PwP } & \multicolumn{2}{|c|}{ Pw/oP } & \multirow{2}{*}{ Statistics } & \multicolumn{2}{|c|}{ PwP } & \multicolumn{2}{|c|}{ Pw/oP } & \multirow{2}{*}{ Statistics } \\
\hline & Average & SD & Average & SD & Average & SD & & Average & SD & Average & SD & \\
\hline All medications & 6.15 & 2.5 & 7.12 & 1.95 & 2.95 & 1.08 & $\begin{array}{l}z=6.5593 \\
p<0.0001\end{array}$ & 9.39 & 1.91 & 5.22 & 1.81 & $\begin{array}{l}z=5.8390 \\
p<0.0001\end{array}$ \\
\hline $\begin{array}{l}\text { Long-term } \\
\text { Medications }\end{array}$ & 3.12 & 2.15 & 3.63 & 2.17 & 1.47 & 0.96 & $\begin{array}{l}z=4.1630 \\
p<0.0001\end{array}$ & 6.39 & 1.58 & 2.19 & 1.15 & $\begin{array}{l}z=-6.4354 \\
p<0.0001\end{array}$ \\
\hline $\begin{array}{l}\text { PRM } \\
\text { medications }\end{array}$ & 1.93 & 1.22 & 2.37 & 1.00 & 0.47 & 0.61 & $\begin{array}{l}z=5.4447 \\
p<0.0001\end{array}$ & 1.94 & 1.16 & 1.92 & 1.25 & $\begin{array}{l}z=-0.0398 \\
p=0.9681\end{array}$ \\
\hline $\begin{array}{l}\text { Symptomatic } \\
\text { medications }\end{array}$ & 2.12 & 1.35 & 2.65 & 1.04 & 0.42 & 0.6 & $\begin{array}{l}z=5.8571 \\
p<0.0001\end{array}$ & 2.28 & 1.74 & 2.08 & 1.36 & $\begin{array}{l}z=-0.4430 \\
p=0.6599\end{array}$ \\
\hline ОTC & 2.42 & 1.83 & 2.92 & 1.30 & 0.79 & 0.79 & $\begin{array}{l}z=5.4224 \\
p<0.0001\end{array}$ & 3.39 & 1.58 & 2.13 & 1.33 & $\begin{array}{l}z=-2.8911 \\
p=0.0039\end{array}$ \\
\hline Prescription drug & 3.73 & 0.43 & 4.21 & 1.80 & 2.16 & 0.69 & $\begin{array}{l}z=4.7983 \\
p<0.0001\end{array}$ & 6.0 & 1.72 & 3.09 & 1.15 & $\begin{array}{l}z=-5.4301 \\
p<0.0001\end{array}$ \\
\hline
\end{tabular}

PRM: pro re nata; OTC: over-the-counter medications

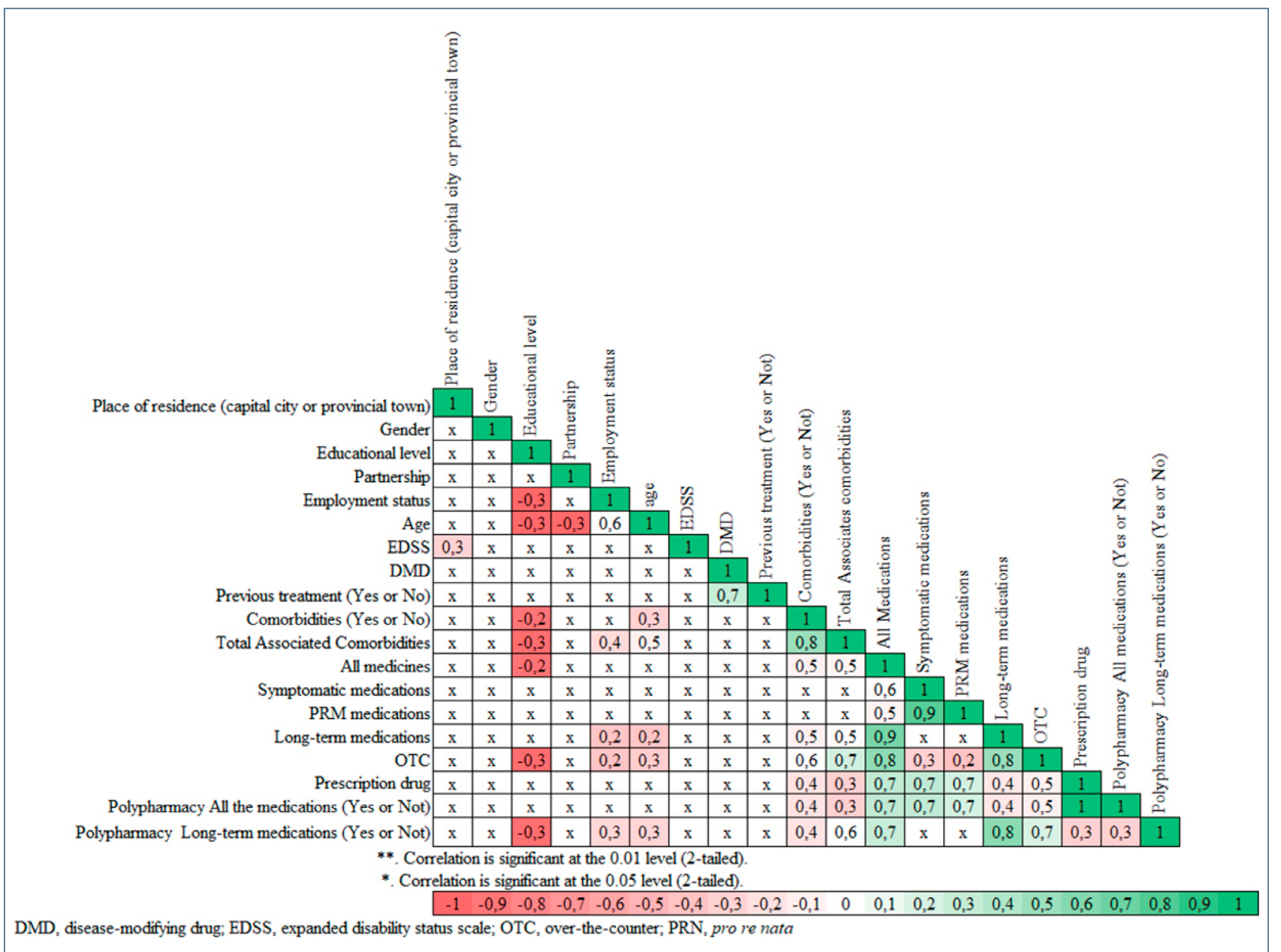

Figure 1: Correlation matrix visualization of the relationship between variables and polypharmacy status. The color gradient represents the degree of pairwise correlation with respect to Pearson's correlation coefficient, while the crosses represent an absence of significance. (DMD: disease-modifying drug; EDSS: Expanded Disability Status Scale; OTC: over-the-counter medications; PRN: pro re nata).

published in 2013, that opined that vitamin D would have no effect on regulating the clinical activity of the disease, acting only to reduce the risk of developing MS, and suggested that further clinical trials were needed in order to rule out any relationship between the use of high doses of vitamin $\mathrm{D}$ and the clinical activity of the disease ${ }^{29}$.

Other studies have also shown that the use of dietary supplements is common in patients with MS and the general population ${ }^{30}$. 
This is due to their easy availability as they require no prescription (OTC) and are affordable. These supplements may be beneficial in the treatment of MS; however, further studies are needed to support their value in the prevention of disease progression ${ }^{31}$.

The presence of cardiovascular diseases contributes to the occurrence of polypharmacy, mainly because of the frequent prescribing of more than one antihypertensive agent. In contrast, gastrointestinal tract drugs, such as proton pump inhibitors, are prescribed to offset the adverse effects of other drug treatments ${ }^{32,33}$.

Drug management plans are needed to optimize treatment, and these should be reviewed regularly in order to identify unnecessary or missing prescriptions, adverse effects, drug interactions, and self-medication. Thus, communication between physicians and pharmacists should be established. Evidence-based, non-medical approaches, such as physiotherapy and behavioral therapies, may offer alternatives or complement pharmacological treatment ${ }^{34}$.

Among the limitations of this study, we can point to the small number of participants. The region studied as being an intermediate or low-incidence zone. Also, it only addressed those patients having regular treatment with DMDs. However, this study provided an overview of the current prevalence and status of medications in this group of patients ${ }^{35}$.

\section{Conclusion}

It is concluded that the presence of comorbidities and age are important factors in the presence of polypharmacy. Further studies of side effects, drug interactions, and adherence problems that demonstrate the role of polypharmacy in MS are needed.

\section{REFERENCES}

1. Multiple Sclerosis International Federation (MSIF). Atlas of MS 2013: mapping multiple sclerosis around the world. London: MSIF, 2013.

2. Belbasis L, Bellou L, Evangelou E, Ioannidis JP, Tzoulaki I. Environmental risk factors and multiple sclerosis: an umbrella review of systematic reviews and meta-analyses. Lancet Neurol. 2015;14(3):263-73.

http://doi.org/10.1016/S1474-4422(14)70267-4

3. Ribeiro TAGJ, Duarte AL, Silva DJ, Borges FE, Costa VM, PapaisAlvarenga RM, et al. Prevalence of multiple sclerosis in Goiânia, Goiás, Brazil. Arq Neuro-Psiquiatr. 2019;77(5):352-6. https://doi.org/10.1590/0004-282x20190032

4. Pedro L, Pais-Ribeiro JL, Pinheiro JP. A importância de um programa de atividade física em doentes com esclerose múltipla na satisfação com a vida e bem-estar psicológico. Saúde Tecnol. 2013;(Supl.)e49-e51.

5. Halpern R, Agarwal S, Dembek C, Borton L, Lopez-Bresnahan M. Comparison of adherence and persistence among multiple sclerosis patients treated with disease-modifying therapies: a retrospective administrative claims analysis. Patient Prefer Adherence. 2011;20(5):73-84.

https://doi.org/10.2147/PPA.S15702

6. Frahm N, Hecker M, Zettl UK. Multi-drug use among patients with multiple sclerosis: A cross-sectional study of associations to clinicodemographic factors. Sci Rep. 2019;9:3743.

https://doi.org/10.1038/s41598-019-40283-5

7. Kochs L, Wegener S, Sühnel A, Voigt K, Zettl UK. The use of complementary and alternative medicine in patients with multiple sclerosis: A longitudinal study. Complement Ther Med. 2014;22(1):166-72.

https://doi.org/10.1016/j.ctim.2013.11.006

8. Richardson K, Ananou A, Lafortune L, Brayne C, Matthews FE. Variation over time in the association between polypharmacy and mortality in the older population. Drugs Aging. 2011;28(7):547-60. https://doi.org/10.2165/11592000-000000000-00000

9. Guthrie B, Makubate B, Hernandez-Santiago V, Dreischulte T. The rising tide of polypharmacy and drug-drug interactions: population database analysis 1995-2010. BMC Med. 2015;13:74. https://doi.org/10.1186/s12916-015-0322-7
10. Maher RL, Hanlon J, Hajiar ER. Clinical consequences of polypharmacy in elderly. Expert Opin Drug Saf. 2014;13(1):57-65. https://doi.org/10.1517/14740338.2013.827660

11. Beiske GAG, Holmøy T, Beiske AG, Johannessen SI, Johannessen Landmark C. Antiepileptic and Antidepressive Polypharmacy in Patients with Multiple Sclerosis. Mult Scler Int. 2015;2015:317859. https://doi.org/10.1155/2015/317859

12. Evans C, Marrie RA, Zhu F, Leung S, LuX, Kingwell E, et al. Adherence to disease-modifying therapies for multiple sclerosis and subsequent hospitalizations. Pharmacoepidemiol Drug Saf. 2017;26(6):702-11. https://doi.org/10.1002/pds.4207

13. Jelinek GA, Weiland TJ, Hadgkiss EJ, Marck $\mathrm{CH}$, Pereira N Medication use in a large international sample of people with multiple sclerosis: associations with quality of life, relapse rate and disability. Neurol Res. 2015;37(8):662-73.

https://doi.org/10.1179/1743132815Y.0000000036

14. Thelen JM, Lynch SG, Bruce AS, Hancock LM, Bruce JM Polypharmacy in multiple sclerosis: relationship with fatigue, perceived cognition, and objective cognitive performance. J Psychosom Res. 2014;76(5):400-4.

https://doi.org/10.1016/j.jpsychores.2014.02.013

15. Laroni A, Signori A, Maniscalco GT, Lanzillo R, Russo CV, Binello E, et al. Assessing association of comorbidities with treatment choice and persistence in MS: A real-life multicenter study. Neurology. 2017;89(22):2222-9.

https://doi.org/10.1212/WNL.0000000000004686

16. Marrie RA, Miller A, Sormani MP, Thompson A, Waubant E, Trojano $\mathrm{M}$, et al. Recommendations for observational studies of comorbidity in multiple sclerosis. Neurology. 2016;86(15):1446-53. https://doi.org/10.1212/WNL.0000000000002474

17. Frahm N, Hecker M, Zettl UK. Polypharmacy in outpatients with relapsing-remitting multiple sclerosis: A single-center study. PLoS One. 2019;24;14(1):e0211120.

https://doi.org/10.1371/journal.pone.0211120

18. Patejdl R, Penner IK, Noack TK, Zettl UK. Multiple sclerosis and fatigue: A review on the contribution of inflammation and immunemediated neurodegeneration. Autoimmun Rev. 2016;15(3):210-20. https://doi.org/10.1016/j.autrev.2015.11.005 
19. Alves CA, Alvarenga MP, Vasconcelos CCF, Batista E, Campanella L, Camargo S, et al. Percalços de um estudo da prevalência de esclerose múltipla no estado do Rio de Janeiro. Rev Bras Neurol Psiquiat. 2013;7(3):103-11.

20. Frahm N, Hecker M, Zettl UK. Polypharmacy in patients with multiple sclerosis: a gender-specific analysis. Biol Sex Differ. 2019;27;10(1):27.

https://doi.org/10.1186/s13293-019-0243-9

21. World Health Organization (WHO). Global age-friendly cities project. Available from: https://www.who.int/ageing/projects/agefriendly_cities.pdf?ua $=1$

22. Marrie R, Reider N, Cohen J, Stuve O, Sorensen PS, Vutter G, et al. A systematic review of the incidence and prevalence of autoimmune disease in multiple sclerosis. Mult Scler. 2015;21(3):282-93. https://doi.org/10.1177/1352458514564490

23. Kaplan TB, Berkowitz AL, Samuels MA. Cardiovascular Dysfunction in Multiple Sclerosis. Neurologist. 2015;20(6):108-14. https://doi.org/10.1097/NRL.0000000000000064

24. Rommer PS, Zettl UK. Managing the side effects of multiple sclerosis therapy: Pharmacotherapy options for patients. Expert Opin Pharmacother. 2018;19(5):483-98.

https://doi.org/10.1080/14656566.2018.1446944

25. Wingerchuk DM, Carter JL. Multiple sclerosis: Current and emerging disease-modifying therapies and treatment strategies. Mayo Clin Proc. 2014;89(2):225-40

https://doi.org/10.1016/j.mayocp.2013.11.002

26. Reich DS, Lucchinetti CF, Calabresi PA. Multiple Sclerosis. N Engl J Med. 2018;378(2): 169-80.

https://doi.org/10.1056/NEJMra1401483

27. Kimball S, Vieth R, Dosch HM, Bar-Or A, Cheung R, Gagne D, et al. Cholecalciferol plus calcium suppresses abnormal PBMC reactivity in patients with multiple sclerosis. J Clin Endocrinol Metab. 2011;96(9):2826-34. https://doi.org/10.1210/jc.2011-0325
28. Brum DG, Comini-Frota ER, Vasconcelos CCF, Dias-Tosta E. Supplementation and therapeutic use of vitamin $D$ in patients with multiple sclerosis: Consensus of the Scientific Department of Neuroimmunology of the Brazilian Academy of Neurology. Arq Neuro-Psiquiatr. 2014;72(2):152-6 https://doi.org/10.1590/0004-282X20130252

29. James E, Dobson R, Kuhle J, Baker D, Giovannoni G, Ramagopalan SV. The effect of vitamin D-related interventions on multiple sclerosis relapses: a meta-analysis. Mult Scler. 2013;19(12): 1571-9

https://doi.org/10.1177/1352458513489756

30. Lentjes MAH. The balance between food and dietary supplements in the general population. Proc Nutr Soc. 2019;78(1):97-109. https://doi.org/10.1017/S0029665118002525

31. Schmitz K, Barthelmes J, Stolz L, Beyer S, Diehl O, Tegeder I. "Disease modifying nutricals" for multiple sclerosis. Pharmacol Ther. 2015;148:85-113 https://doi.org/10.1016/j.pharmthera.2014.11.015

32. Mathur R, Hull SA, Badrick E, Robson J. Cardiovascular multimorbidity: the effect of ethnicity on prevalence and risk factor management. Br J Gen Pract. 2011;61(586):262-70. https://doi.org/10.3399/bjgp11X572454

33. Delcher A, Hily S, Boureau AS, Chapelet G, Berrut G, Decker L. Multimorbidities and overprescription of proton pump inhibitors in older patients. PLoS One. 2015;10(11):e0141779. https://doi.org/10.1371/journal.pone.0141779

34. Wade BJ. Spatial analysis of global prevalence of multiple sclerosis suggests need for an updated prevalence scale. Mult Scler Int. 2014; 2014:124578. https://doi.org/10.1155/2014/124578

35. Broekmans T, Roelants M, Feys P, Alders G, Gijbels D, Hanssen I, et al. Effects of long-term resistance training and simultaneous electro-stimulation on muscle strength and functional mobility in multiple sclerosis. Mult Scler. 2011;17(4):468-77. https://doi.org/10.1177/1352458510391339 